\title{
Acute pancreatitis patient registry to examine novel therapies in clinical experience (APPRENTICE): an international, multicenter consortium for the study of acute pancreatitis
}

\author{
Georgios I. Papachristoua, Jorge D. Machicadoa, Tyler Stevens ${ }^{b}$, Mahesh Kumar Goenkac, \\ Miguel Ferreirad, Silvia C. Gutierreze, Vikesh K. Singhf, Ayesha Kamal', Jose A. Gonzalez-Gonzalez ${ }^{\mathrm{f}}$, \\ Mario Pelaez-Luna ${ }^{h}$, Aiste Gulla ${ }^{i, j}$, Narcis O. Zarnescuk, Konstantinos Triantafyllou', Sorin T. Barbum, \\ Jeffrey Easlern, Carlos Ocampo ${ }^{\circ}$, Gabriele Capurso ${ }^{p}$, Livia Archibugi ${ }^{p}$, Gregory A. Cote ${ }^{q}$, Louis Lambiaser, \\ Rakesh Kochhar', Tiffany Chuab, Subhash Ch. Tiwari', Haq Nawaz', Walter G. Park", Enrique de-Madariav, \\ Peter J. Leew, Bechien U. Wux, Phil J. Greera, Mohannad Duguma ${ }^{a}$, Efstratios Koutroumpakis ${ }^{a}$, Venkata \\ Akshintalaf, Amir Gougola for the APPRENTICE Study
}

University of Pittsburgh Medical Center, Pittsburgh, Pennsylvania, USA; Cleveland Clinic Foundation, Cleveland, Ohio, USA; Apollo Gleneagles Hospitals Kolkata, India; Hospital Nacional de Itauguá, Paraguay; Hospital Nacional "Profesor Alejandro Posadas", Buenos Aires, Argentina; Johns Hopkins Medical Institutions, Baltimore, Maryland, USA; Universidad Autónoma de Nueva León, Monterrey, Mexico; Instituto Nacional de Ciencias Médicas y Nutrición Salvador Zubirán - Universidad Autónoma de Mexico, Mexico City, Mexico; Georgetown University Hospital, Washington, DC, USA; Lithuanian University of Health Sciences, Kaunas, Lithuania; University Emergency Hospital Bucharest, "Carol Davila" University of Medicine and Pharmacy, Bucharest, Romania; Attikon University General Hospital, Athens, Greece; University of Medicine and Pharmacy "Iuliu Hatieganu", Cluj-Napoca, Romania; Indiana University School of Medicine, Indianapolis, Indiana, USA; Hospital General de Argudos "Dr. Cosme Argerich", Buenos Aires, Argentina; S. Andrea Hospital, Sapienza University of Rome, Italy; Medical University of South Carolina, Charleston, South Carolina, USA; University of Tennessee College of Medicine, Chattanooga, Tennessee, USA; Postgraduate Institute of Medical Education and Research, Chandigarh, India; Eastern Maine Medical Center, Maine, Bangor, USA; Stanford University, Stanford, Kaiser Permanente, Pasadena, California, USA; Hospital General Universitario de Alicante, Institute de Investigación Sanitaria y Biomédica de Alicante (ISABIAL - Fundación FISABIO), Alicante, Spain; University Hospitals Cleveland Medical Center, Ohio, USA

\footnotetext{
${ }^{a}$ Division of Gastroenterology and Hepatology, University of Pittsburgh Medical Center, Pittsburgh, Pennsylvania, USA (Georgios I. Papachristou, Jorge D. Machicado, Phil J. Greer, Mohannad Dugum, Efstratios Koutroumpakis Amir Gougol); 'Cleveland Clinic Foundation, Cleveland, Ohio, USA (Tyler Stevens, Tiffany Chua); 'Apollo Gleneagles Hospitals Kolkata, Kolkata, India (Mahesh Kumar Goenka, Subhash Ch.

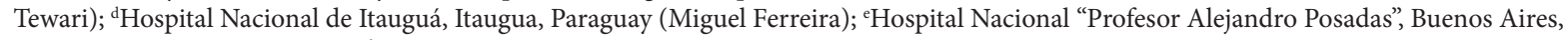
Argentina (Silvia C. Gutierrez); 'Johns Hopkins Medical Institutions, Baltimore, Maryland, USA (Vikesh K. Singh Ayesha Kamal, Venkata

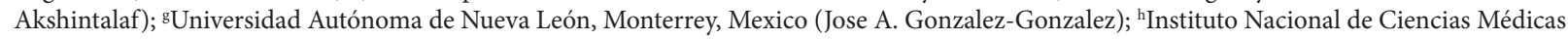
y Nutrición Salvador Zubirán-Universidad Autónoma de Mexico, Mexico City, Mexico (Mario Pelaez-Luna); iGeorgetown University Hospital,

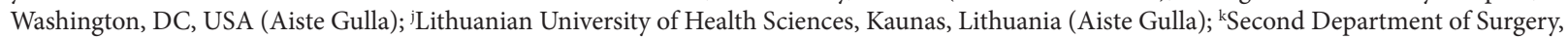
University Emergency Hospital Bucharest, "Carol Davila" University of Medicine and Pharmacy, Bucharest, Romania (Narcis O. Zarnescu); ${ }^{1}$ Attikon University General Hospital, Athens, Greece (Konstantinos Triantafyllou); ${ }^{m}$ University of Medicine and Pharmacy "Iuliu Hatieganu”, Cluj-Napoca, Romania (Sorin T. Barbu); "Indiana University School of Medicine, Indianapolis, Indiana, USA (Jeffrey Easler); ${ }^{\circ}$ Hospital General de Argudos "Dr. Cosme Argerich", Buenos Aires, Argentina (Carlos Ocampo); PDigestive and Liver Disease Unit, S. Andrea Hospital, Sapienza University of Rome, Rome, Italy (Gabriele Capurso, Livia Archibugi); ${ }^{9}$ Medical University of South Carolina, Charleston, South Carolina, USA (Gregory A.

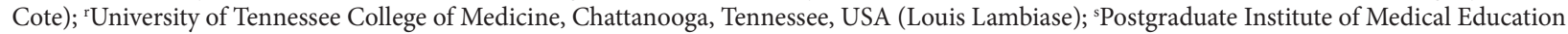
and Research, Chandigarh, India (Rakesh Kochhar); 'Eastern Maine Medical Center, Maine, Bangor, USA (Haq Nawaz); "Stanford University,

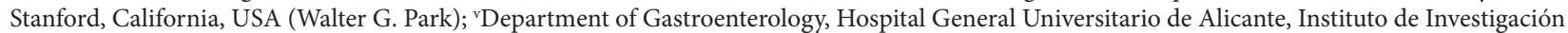
Sanitaria y Biomédica de Alicante (ISABIAL - Fundación FISABIO), Alicante, Spain (Enrique de-Madaria); 'Wniversity Hospitals Cleveland Medical Center, Ohio, USA (Peter J. Lee); Kaiser Permanente, Pasadena, California, USA (Bechien U. Wu)
}

Conflict of Interest: None

Correspondence to: Georgios I. Papachristou, MD, PhD, Professor of Medicine, Division of Gastroenterology and Hepatology, University of Pittsburgh Medical Center, 200 Lothrop Street, M2, C-wing, Pittsburgh, PA 15213, USA, Tel.: +1 412647 8132, Fax: +1 4126471880 , e-mail: papachri@pitt.edu

Received 10 October 2016; accepted 1 November 2016; published online 01 December 2016

DOI: https://doi.org/10.20524/aog.2016.0109 


\section{Abstract}

Background We have established a multicenter international consortium to better understand the natural history of acute pancreatitis (AP) worldwide and to develop a platform for future randomized clinical trials.

Methods The AP patient registry to examine novel therapies in clinical experience (APPRENTICE) was formed in July 2014. Detailed web-based questionnaires were then developed to prospectively capture information on demographics, etiology, pancreatitis history, comorbidities, risk factors, severity biomarkers, severity indices, health-care utilization, management strategies, and outcomes of AP patients.

Results Between November 2015 and September 2016, a total of 20 sites (8 in the United States, 5 in Europe, 3 in South America, 2 in Mexico and 2 in India) prospectively enrolled 509 AP patients. All data were entered into the REDCap (Research Electronic Data Capture) database by participating centers and systematically reviewed by the coordinating site (University of Pittsburgh). The approaches and methodology are described in detail, along with an interim report on the demographic results.

Conclusion APPRENTICE, an international collaboration of tertiary AP centers throughout the world, has demonstrated the feasibility of building a large, prospective, multicenter patient registry to study AP. Analysis of the collected data may provide a greater understanding of AP and APPRENTICE will serve as a future platform for randomized clinical trials.

Keywords Acute pancreatitis, international multicenter consortium, methodology, APPRENTICE

Ann Gastroenterol 2017; 30 (1): 1-8

\section{Introduction}

The annual global incidence of acute pancreatitis (AP) ranges from 13 to $45 / 100,000$ and has increased substantially over time across nations [1]. As a consequence, the number of AP-related hospital admissions has doubled in the past 3 decades, and AP is now one of the leading causes of gastrointestinal-related hospitalizations, with direct annual costs of up to US\$2.6 billion in the US [2-4].

The implementation in clinical practice of early and goal-directed fluid resuscitation, intensive care for organ failure, nutritional optimization, antibiotics that penetrate pancreatic tissue, minimally invasive techniques for pancreatic debridement, and a "step-up" approach, has likely contributed to a decrease in AP-related case fatality over the last 40 years $[1,3]$. Several pharmacologic therapies have been tested in randomized controlled trials and have failed to show any significant benefit in the management of AP [5-8]. Investigation of other promising experimental treatments has been confined to pre-clinical and small clinical studies [9-12]. As a consequence, no specific drug therapy is available to mitigate local and systemic complications in AP [13]. With the discovery of novel molecular targets in the pathogenesis of AP, the advent of new experimental disease-specific drugs is expected [14]. Therefore, a collaborative, multicenter network is a fundamental requirement for translating experimental drug development into large-scale randomized controlled trials.

With the goal of serving as a platform to examine novel therapies for AP, we have organized the "Acute Pancreatitis Patient Registry to Examine Novel Therapies in Clinical
Experience" (APPRENTICE). To move toward this objective, we have recognized the importance of creating an electronic prospective registry from high-volume international centers with expertise in pancreatic diseases, in order to describe the current trends of demographics, risk factors, clinical profile, treatment patterns, and outcomes of AP around the world. In addition, the establishment of this unique international collaboration will allow us to use it as a platform for future randomized clinical trials that can translate new therapies in less time and with a diversified patient population. It could also serve as a repository for proteomic and genomic data in patients with AP.

Herein, we describe the study methodology and report preliminary demographic results from the cohort.

\section{Materials and methods}

\section{Development of APPRENTICE and participating centers}

The idea of starting an international prospective registry in AP was developed during the AP working group at PancreasFest 2014 (July 2014, Pittsburgh, PA, USA) under the sponsorship of the Collaborative Alliance for Pancreatic Education and Research (CAPER). APPRENTICE members have since maintained collaborative communications via e-mail, teleconferences (webinars), and face-to-face meetings at annual conferences of gastrointestinal societies. Expertise was sought from APPRENTICE members and founders of other pancreatitis multicenter research consortia (NAPS2 and 
INSPPIRE) $[15,16]$. The University of Pittsburgh has led this initiative from the beginning and serves as the coordinating center. An analysis and publication committee of 5 members from four different continents has been established. This committee is in charge of collecting research ideas, setting deadlines, and deciding on the authorship of future publications. Finally, a strategy has been developed to apply for grant funding in the near future.

The initial requirements for a center to participate in APPRENTICE include a letter from their local Institutional Board Review (IRB) or Ethics Committee and a signed datause agreement form to be submitted to the University of Pittsburgh, which serves as the "umbrella" IRB. To date, 20 tertiary referral centers with established expertise in pancreatic disorders from 9 countries have joined the consortium.

\section{Subject recruitment}

Standardized inclusion and exclusion criteria have been developed to ensure the uniformity of the study population. The diagnosis of AP is based on the presence of at least 2 of the following 3 features: abdominal pain characteristic of AP, serum amylase and/or lipase at least 3 times the upper limit of normal, and characteristic findings of AP on abdominal imaging. To be eligible, participants must be adults ( $\geq 18$ years old), willing to participate, and enrolled within 7 days from symptom onset. Patients with AP following trauma, a history of chronic pancreatitis, organ transplantation, pancreatic cancer, or any type of cancer that required chemotherapy/ radiation in the past year, are excluded. Subjects fulfilling the entry criteria at the study sites are offered participation by the site investigator. Prior to enrollment, study subjects sign an informed consent form based on local IRB requirements.

Site investigators are responsible for identifying patients with AP during their hospital admission. The methodology for prompt early detection of candidates may vary from center to center. Thus, the APPRENTICE prospective cohort likely represents a fraction of all consecutive patients admitted to each center, depending on the availability of resources at each site.

\section{Development of questionnaires}

An initial draft of the study questionnaires was prepared in the autumn of 2014. This was opened to comments and feedback from the entire consortium in monthly webinars. Subsequently, during a 4-month pilot phase (November 2015-February 2016), the study questionnaires were further updated until a final version was approved before the main phase of the study was started. Information on demographics, etiology, pancreatitis history, comorbidities, clinical characteristics, early prognostic markers, severity classification, healthcare utilization, treatment strategies, and hospital course has been organized into 260 variables. Anonymized data are collected prospectively by the site investigator or research coordinator, based on interactions with the patient and a review of the medical records.

In addition, enrolled patients are contacted within 60120 days after hospital discharge to complete a follow-up questionnaire, adding approximately 30 more variables to the registry. This takes place during a clinic visit or through a telephone call. The follow-up questionnaire mainly focuses on recurrent attacks of AP, the need for delayed intervention, and the potential development of AP-related complications.

\section{Questionnaires}

a) Demographics, past history, and etiology

Demographic information includes age, sex, race, weight, height, body mass index, and waist circumference measurement. Smoking history is classified as never smoker $(<100$ cigarettes or 5 packs in lifetime), former smoker ( $>6$ months without smoking), and current smoker (within the last 6 months). The total years of smoking and the average number of cigarettes per day are recorded. Alcohol consumption is classified as never ( $<20$ drinks in lifetime), former ( $>6$ months without drinking), and current (within the last 6 months). In addition, the average number of drinks on a drinking day, the average drinking days per week, the total years of alcohol consumption, and the date of last drink are obtained.

In the section on personal history, information about the prior history of AP is recorded, including number of episodes, baseline triglyceride levels, and cholecystectomy status. The burden of comorbidities is measured using the Charlson Comorbidity Index [17]. Information is sought regarding any first-degree relative with a history of AP, chronic pancreatitis, or cystic fibrosis. Any use of statins, non-steroid anti-inflammatory drugs within the last week, or other recently started medications is also recorded.

The dates of symptom onset and initial hospital admission are recorded. If the patient is transferred from another hospital, the date of transfer is also registered. The primary etiology of AP is classified as biliary, alcoholic, idiopathic, hypertriglyceridemic, subsequent to endoscopic retrograde cholangiopancreatography (ERCP), or other. A secondary etiology of AP is recorded in selected cases when 2 causes coexist. For example, in patients with alcoholic or biliary AP who also have hypertriglyceridemia, hypertriglyceridemia is deemed to be the secondary etiology. In addition, the timing with which triglycerides are measured in relation to the disease onset, and their highest level within the first $48 \mathrm{~h}$ after presentation are recorded.

b) Biomarkers of severity, hospital admission and outcomes Vital signs, pain intensity assessment based on a visual scale, physical findings, and laboratory markers (white cell count, hematocrit, creatinine, blood urea nitrogen, lipase level) are obtained upon initial hospital admission, and after 1, 2, and 7 days. In patients who meet the criteria for systemic inflammatory response syndrome (SIRS), the timing of onset and the duration of SIRS ( $<48$ vs. $\geq 48 \mathrm{~h}$ ) are also entered. 
Admission to the intensive care unit (ICU), in addition to the reason for ICU admission, the length of stay, and death while in the ICU are recorded. The total length of hospital stay and in-hospital mortality are also entered. The cause of death is classified as either unrelated or related to pancreatitis. The patients' perspective on the hospital stay is assessed by asking how often they are treated with courtesy and respect, and how often their pain is controlled. Possible answers are never, sometimes, usually, and always.

The severity of AP is defined based on the revised Atlanta classification [18] and the determinant-based classification [19]. The definition of organ failure used by the modified Marshall scoring system is used [20]. The timing of the onset of organ failure, the first failing system(s), and the duration of organ failure are recorded. Data on cardiovascular failure (systolic blood pressure $<90 \mathrm{mmHg}$ not fluid responsive, $\mathrm{pH}<7.3$, or use of inotropes), respiratory failure $\left(\mathrm{PaO}_{2} / \mathrm{FiO}_{2}<300\right.$, or the need for intubation), and renal failure (serum $\mathrm{Cr}>1.8 \mathrm{mg} / \mathrm{dL}$ or $>169 \mu \mathrm{mol} / \mathrm{L}$, or the need for hemodialysis) are recorded. Use of contrast-enhanced computed tomography (CT) during the hospital admission or within 1 month of follow up is recorded. The CT performed closest to the seventh day of admission is entered to classify the pancreatic findings as normal, interstitial, or necrotizing AP. The extent of pancreatic necrosis $(<30 \%, 30-50 \%,>50 \%)$ and findings of peripancreatic necrosis are included. Whether pancreatic necrosis evolves into walled-off necrosis is also recorded, with precise timing of the finding and the largest diameter of the collection in $\mathrm{cm}$. Any diagnosis of infected pancreatic necrosis and extra-pancreatic infection during the hospitalization is noted.

c) Early and delayed management

Data on intravenous fluids early during hospital admission are recorded. The type and volume (total in $\mathrm{mL}$ ) of intravenous fluid received within first 6 and $24 \mathrm{~h}$ are entered. Opioid use, route of administration, type of opioid, dose of opioid and other analgesic use within the first, second, third, and seventh days of initial hospital admission is recorded. The total days of opioid used are also entered. The type, route, timing, and tolerance of the initial feeding attempt are recorded. A similar approach is followed for the second and third feeding routes. Oral tolerance is defined as patients who tolerated oral feedings for more than $24 \mathrm{~h}$.

Pancreatic interventions are classified as early (within 2 weeks from initial admission) and late (after 2 weeks from initial admission). Data are organized by the type (drainage or drainage/debridement) and mode (laparotomy, minimally invasive surgery, percutaneous, and endoscopic) of intervention. Data on early and delayed interventions are recorded. Data on ERCP or cholecystectomy during the hospital admission are entered, as well as the indication for ERCP. The date of each intervention is entered systematically.

d) Follow up

Efforts are made to contact the enrolled patients close to 90 days after hospital discharge for follow up. Pain, pain intensity (based in a visual scale), pain frequency (number of days in last 7 days), and nausea/vomiting within the last
7 days from the day of contact are recorded. Changes on smoking and drinking habits are measured. Patients are asked whether they continue drinking alcohol, the average number of drinks on a drinking day, and the number of days that they drink on a week. In addition, whether they continue smoking, and the number of cigarettes per day is also recorded.

To assess the clinical course at follow up, patients are asked whether they required emergency visits or hospitalizations due to pancreatitis symptoms, or if they developed a documented recurrent attack of AP, new-onset diabetes, or overt steatorrhea. Use of medications such as oral antidiabetics, insulin, and pancreatic enzymes is also assessed. Patients are also asked if they underwent cholecystectomy after the hospital discharge.

\section{Database management and study initiation}

All the data are prospectively recorded and stored in REDCap (Research Electronic Data Capture) by study personnel at each participating center. REDCap is a frequently used program for clinical, basic, and translational research [16,21]. This novel software allows collaborative access to data across different centers around the world. To ensure confidentiality, each subject's information is anonymized and assigned a study code before entry into REDCap. Every electronic form is accessible only to IRB-approved research team members, who have a username and password for the web application. Each entry field contains real-time validation codes, integrity checks, and other mechanisms to ensure data quality.

Data are stored centrally in the REDCap coordinating site at the University of Pittsburgh. A data quality monitoring team at the coordinating center verifies the quality and the completeness of the data, identifies discrepancies in entered data, eliminates duplicate patient entries, and provides feedback to participating centers so as to optimize data entry.

After submission of a request for the database service to REDCap, a working web-based electronic data collection application was developed for the project. Using this prototype application, the metadata spreadsheet was tested during a pilot phase that involved 10 centers ( 9 domestic and 1 international). In this 4-month phase, 57 patients were enrolled to test every aspect of the data collection process. Starting April 2016, the REDCap system has been advanced to production status for the main phase, aiming to prospectively enroll at least $1000 \mathrm{AP}$ patients. An outline of the steps of APPRENTICE, from study conception to study initiation is presented in Fig. 1.

REDCap offers several other advantages. While it facilitates some statistical analysis, it also allows the export of data for use with common statistical packages. It provides a file repository for storing and sharing documents (e.g. study protocol). There is also a data dictionary available for researchers. Finally, this software is free of charge, which allows an adequate platform for unfunded investigator-initiated research projects like APPRENTICE.

The APPRENTICE analysis and publication committee has developed authorship guidelines, which have been approved 
by all participating centers. Site investigators who want to use the registry to answer a specific research question have been submitting their proposals to the analysis and publication committee. After obtaining approval, they will proceed with the project once enrollment is completed. The number of patients recruited by center will be taken into consideration during project assignment. Investigators assigned a project will be required to have a manuscript ready for publication within 6 months.

\section{Results}

A total of 509 subjects ( $52 \%$ women) have been recruited from November 2015 to September 2016. The distribution of patients per site is shown in Fig. 2.

Demographics and disease etiology in all the study participants and by continent are shown in Table 1 . The mean age of participants is 51 years $(S D=19$, median 50). Participants are older in European centers $(58 \pm 18$ years) and younger in Asia ( $45 \pm 14$ years). About half (47\%) of the population is white and a third (33\%) is Hispanic.
The leading cause of AP in all the continents is biliary (46\%), but the proportion varies widely, from $26 \%$ in US to $82 \%$ in South America. Idiopathic is the second most common etiology (17\%), followed by alcoholic (14\%), post-ERCP (12\%), and hypertriglyceridemic pancreatitis (5\%). Alcoholic etiology is less frequent in South America (0\%) and Mexico (6\%), compared with Europe (20\%) and Asia (32\%). Moreover, postERCP pancreatitis is the second most common cause of AP in the US (24\%), whereas it is infrequent in other continents. Both idiopathic and hypertriglyceridemic pancreatitis appear to have a homogeneous distribution across continents. The proportion of participants with a sentinel attack of AP is $75 \%$, with more cases of recurrent AP enrolled in the US (39\%).

\section{Discussion}

There are several examples of international collaborations that have contributed to expanding the field of AP. Two different groups, the AP Classification Working group and the Pancreatitis Across Nations Clinical Research and Education Alliance (PANCREA) have recently developed two

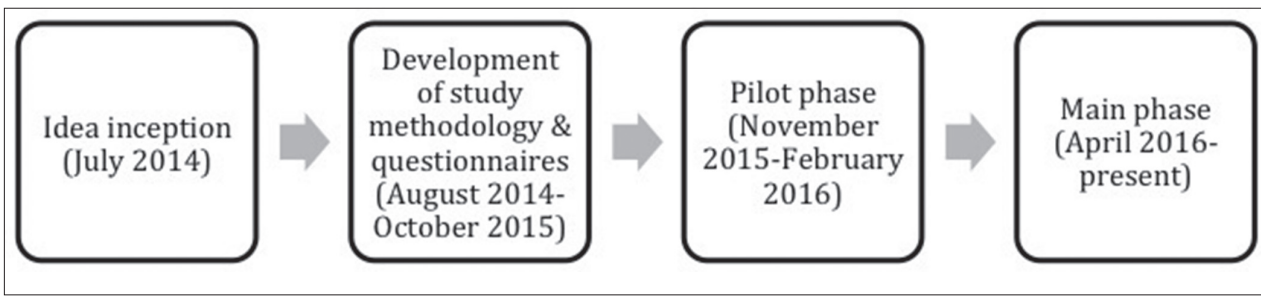

Figure 1 APPRENTICE methodology flowchart

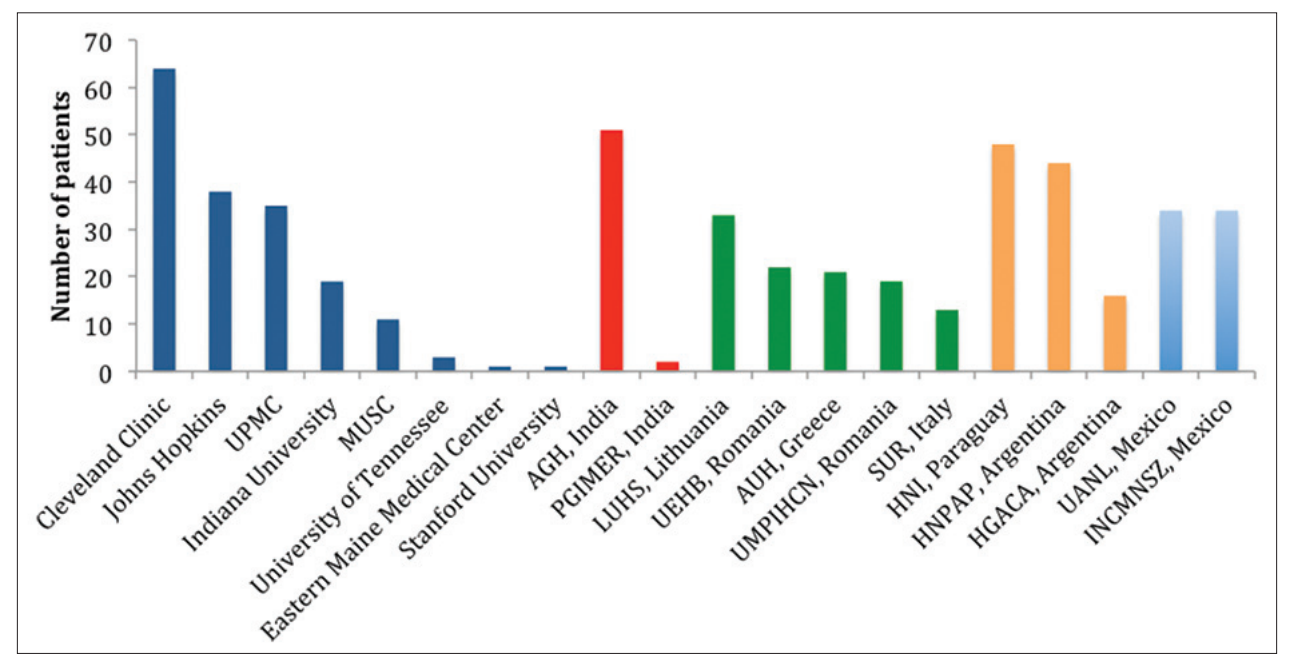

Figure 2 Distribution of patients enrolled across APPRENTICE centers

UPMC, University of Pittsburgh Medical Center; MUSC, Medical University of South Carolina; AGH, Apollo Gleneagles Hospital, India; PGIMER, Postgraduate Institute of Medical Education and Research, India; LUHS, Lithuanian University of Health Sciences, Lithuania; UEHB, University Emergency Hospital Bucharest, Romania; AUH, Attikon University Hospital, Greece; UMPIHCN, University of Medicine and Pharmacy "Iuliu Hatieganu" Cluj-Napoca, Romania; SUR, Sapienza University of Rome, Italy; HNI, Hospital Nacional de Itauguá, Paraguay; HNPAP, Hospital Nacional "Profesor A. Posadas", Argentina; HGADCA, Hospital General de Agudos "Dr. Cosme Argerich", Argentina; UANL, Universidad Autónoma de Nueva León, Mexico; INCMNSZ, Instituto Nacional de Ciencias Médicas y Nutrición Salvador Zubirán, Mexico 
Table 1 Preliminary analysis of the distribution of demographics and etiology of AP in each of the continents from the APPRENTICE registry

\begin{tabular}{|c|c|c|c|c|c|c|}
\hline Variables & All & US & Europe & South America & Mexico & Asia \\
\hline Number of centers & 20 & 8 & 5 & 3 & 2 & 2 \\
\hline Number of subjects & 509 & 172 & 108 & 108 & 68 & 53 \\
\hline Mean age $( \pm S D)$ & $51 \pm 19$ & $51 \pm 18$ & $58 \pm 18$ & $48 \pm 19$ & $47 \pm 20$ & $45 \pm 14$ \\
\hline Sex (\% males) & 49 & 48 & 56 & 38 & 37 & 74 \\
\hline \multicolumn{7}{|l|}{ Race, $\%$} \\
\hline White & 47 & 70 & 98 & 11 & 0 & 0 \\
\hline Hispanic & 33 & 2 & 1 & 89 & 100 & 0 \\
\hline Asian & 12 & 3 & 1 & 0 & 0 & 100 \\
\hline Black & 8 & 25 & 0 & 0 & 0 & 0 \\
\hline \multicolumn{7}{|l|}{ Etiology, \% } \\
\hline Gallstone & 46 & 26 & 48 & 82 & 53 & 36 \\
\hline Alcoholic & 14 & 16 & 20 & 0 & 6 & 32 \\
\hline Idiopathic & 17 & 21 & 17 & 8 & 17 & 17 \\
\hline HyperTG & 5 & 5 & 7 & 2 & 11 & 4 \\
\hline Post-ERCP & 12 & 24 & 3 & 6 & 5 & 4 \\
\hline Other & 6 & 8 & 5 & 2 & 8 & 7 \\
\hline \multicolumn{7}{|l|}{ History of AP, \% } \\
\hline First episode & 75 & 61 & 81 & 87 & 78 & 77 \\
\hline Recurrent & 25 & 39 & 19 & 13 & 22 & 23 \\
\hline
\end{tabular}

severity classification systems that have changed the current landscape of AP $[18,19]$. For decades, worldwide experts in the pancreas field have attempted to reunite and propose recommendations for the management of different aspects of AP [22-24]. However, with only a few exceptions, international collaborations have not led to original prospective studies or randomized controlled trials [25].

Constructing an international prospective registry of wellphenotyped AP patients is the first step towards our goal of systematically characterizing AP throughout the world and advancing the management of AP. This strategy will provide information on care needs, therapies delivered and outcomes in real-world practice. The advantages of an international rather than a local approach include better external validity, and a larger study population within a shorter period of time. We have established the feasibility of collaboration among 20 centers spread over 9 countries and 4 continents. Collaborative academic researchers will be able to use the final database as a powerful research tool to answer clinical questions.

A similar approach has been taken by the INSPPIRE group to advance the understanding of pediatric pancreatitis [16]. Their initial work has yielded several lessons useful for other international pancreatitis collaborations. Similarly to our study, it was unfunded at the beginning, but funding from the National Institutes of Health was subsequently secured for further research. REDCap has been used successfully as their electronic data capture method, demonstrating its utility in multicenter pancreas research studies. Their prospective data from around 300 children have served to advance our understanding of the current status of pediatric pancreatitis, with published reports in high-impact journals [26,27].

Conducting large-scale prospective randomized trials can be tedious and expensive. Consequently, owing to both the cost and the regulatory burden, many new and existing interventions for AP have not been evaluated rigorously, and the most influential trials have enrolled limited numbers of participants from certain geographical areas [28-30]. Registrybased randomized clinical trials have become a powerful tool for increasing the efficiency and cost-effectiveness of clinical trials [31]. By including a randomization module in a large prospective registry, with unselected consecutive enrollment, important features of a randomized controlled trial can be combined with the inclusiveness and efficiency of a large prospective clinical registry. Benefits of the linkage to a registry might include the capacity to identify eligible patients, to enroll larger patient populations, and to conduct long-term follow up at low cost. In addition, developing a registry will build the infrastructure within participating centers for the early identification and rapid enrollment of patients admitted with AP.

To our knowledge, this is the first international prospective registry of adult patients with AP. Furthermore, there is no previous experience from prospective registry-based randomized trials in the field of pancreatic disorders. Therefore, our platform may serve as an innovative strategy to translate novel therapies, discover new biomarkers for the prediction of 
severity, and create an international bank of proteomics and genomic information.

However, APPRENTICE has limitations. First, the different distribution of etiologies between continents is difficult to interpret from our preliminary data. This may be a consequence of intrinsic differences in demographics and risk exposures between populations. Selection and referral bias are also possible, as we recruit patients from tertiary care centers. Consecutive enrollment has been challenging, especially in centers that do not have an automatic or well-established method for identifying AP patients during hospital admission. Therefore, the distribution of etiologies seen in our cohort may not match the distribution of all patients admitted to each site during the study period, and our cohort may represent a random sampling of patients with AP in our practices rather than a true longitudinal cohort. Some of these considerations may explain, for example, why higher rates of post-ERCP pancreatitis are observed in the US compared with other continents. A validation of the APPRENTICE cohort will be needed for the interpretation of future reports. Second, even though the questionnaires were refined by experts during the pilot phase, they may not capture some important confounding factors, outcomes or details in interventions. Third, collecting information in an electronic database, entered by different research personnel, can be problematic. The quality of the data entered depends on the study coordinators entering complete and reliable information for every patient. We have worked on minimizing this problem and ensuring high data quality by giving training to investigators entering data, performing a 4-month pilot period of data collection, addressing issues regularly through webinars, and verifying the quality and completeness of data periodically by the monitoring group.

\section{Summary Box}

\section{What is already known:}

- No specific drug therapy is available to mitigate complications of acute pancreatitis (AP)

- A collaborative, multicenter network is fundamental for translating experimental drug development in AP into randomized controlled trials

\section{What the new findings are:}

- APPRENTICE is an international collaboration that has successfully enrolled more than 500 patients with AP from four different continents

- This prospective registry will provide information on epidemiologic trends, current therapies, care needs, and outcomes of AP in real-world practice

- APPRENTICE will serve as a future platform for randomized clinical trials
In summary, APPRENTICE has demonstrated to be a robust and effective international collaboration, extending to four different continents, that has been successfully enrolling patients with AP worldwide. Future research using this platform will provide information on epidemiologic trends, current therapies, care needs, and outcomes between geographical regions in real-world practice. Furthermore, APPRENTICE may prove to be critical in developing personalized medicine approaches, and integrating randomized clinical trials of novel therapies in AP.

\section{Acknowledgments}

We greatly appreciate the contributions of Carl Manzo, John Perlegos, Anwar Dudekula, Dhiraj Yadav, and David C. Whitcomb from the University of Pittsburgh Medical Center, Pittsburgh, Pennsylvania; Kunjam Modha from the Cleveland Clinic Foundation, Cleveland, Ohio; Julio Ramírez Sotomayor from the Hospital Nacional de Itauguá, Itaugua, Paraguay; Laura Marchi, Lorena Perna, Juan Codd, Ana Zambrana, and Indira Gabo from the Hospital Nacional "Profesor Alejandro Posadas", Buenos Aires, Argentina; David Gomez Ortiz from the Universidad Autónoma de Nueva León, Monterrey, Mexico; Jorge Hernandez from the Instituto Nacional de Ciencias Médicas y Nutrición Salvador Zubirán - Universidad Autónoma de Mexico, Mexico City, Mexico; Zilvinas Dambrauskas from the Lithuanian University of Health Sciences, Kaunas, Lithuania; Chronis Gatopoulos from Attikon University General Hospital, Athens, Greece; Tudor Cerciu and Nicoleta Pers from the University of Medicine and Pharmacy "Iuliu Hatieganu", Cluj-Napoca, Romania; Suzette Schmitt from Indiana University School of Medicine, Indianapolis, Indiana; Zandalazini Hugo from the Hospital General de Argudos "Dr. Cosme Argerich", Buenos Aires, Argentina; Samreen Fathima, Cynthia Whited and Denise Michaud from Eastern Maine Medical Center, Maine, Bangor; and April Williams, and Emily Depue from the Medical University of South Carolina, Charleston, South Carolina.

\section{References}

1. Yadav D, Lowenfels AB. Trends in the epidemiology of the first attack of acute pancreatitis: a systematic review. Pancreas 2006;33:323-330.

2. Goldacre MJ, Roberts SE. Hospital admission for acute pancreatitis in an English population, 1963-98: database study of incidence and mortality. BMJ 2004;328:1466-1469.

3. Munigala S, Yadav D. Case-fatality from acute pancreatitis is decreasing but its population mortality shows little change. Pancreatology 2016;16:542-550.

4. Peery AF, Crockett SD, Barritt AS, et al. Burden of gastrointestinal, liver, and pancreatic diseases in the United States. Gastroenterology 2015;149:1731-1741.e3.

5. Johnson CD, Kingsnorth AN, Imrie CW, et al. Double blind, randomised, placebo controlled study of a platelet activating factor 
antagonist, lexipafant, in the treatment and prevention of organ failure in predicted severe acute pancreatitis. Gut 2001;48:62-69.

6. Dürr HK, Maroske D, Zelder O, Bode JC. Glucagon therapy in acute pancreatitis. Report of a double-blind trial. Gut 1978;19:175-179.

7. Büchler M, Malfertheiner P, Uhl W, et al. Gabexate mesilate in human acute pancreatitis. German Pancreatitis Study Group. Gastroenterology 1993;104:1165-1170.

8. Uhl W, Büchler MW, Malfertheiner P, Beger HG, Adler G, Gaus W. A randomised, double blind, multicentre trial of octreotide in moderate to severe acute pancreatitis. Gut 1999;45:97-104.

9. Schneider L, Jabrailova B, Soliman H, et al. Pharmacological cholinergic stimulation as a therapeutic tool in experimental necrotizing pancreatitis. Pancreas 2014;43:41-46.

10. Yildirim AO, Ince M, Eyi YE, et al. The effects of glycyrrhizin on experimental acute pancreatitis in rats. Eur Rev Med Pharmacol Sci 2013;17:2981-2987.

11. Gulcubuk A, Haktanir D, Cakiris A, et al. Effects of curcumin on proinflammatory cytokines and tissue injury in the early and late phases of experimental acute pancreatitis. Pancreatology 2013;13:347-354.

12. Lampropoulos P, Lambropoulou M, Papalois A, et al. The role of apigenin in an experimental model of acute pancreatitis. J Surg Res 2013; 183:129-137.

13. Afghani E, Pandol SJ, Shimosegawa T, et al. Acute pancreatitisprogress and challenges: a report on an International Symposium. Pancreas 2015;44:1195-1210.

14. Gukovskaya AS, Pandol SJ, Gukovsky I. New insights into the pathways initiating and driving pancreatitis. Curr Opin Gastroenterol 2016 Jul 13. [Epub ahead of print].

15. Whitcomb DC, Yadav D, Adam S, et al; North American Pancreatic Study Group. Multicenter approach to recurrent acute and chronic pancreatitis in the United States: the North American Pancreatitis Study 2 (NAPS2). Pancreatology 2008;8:520-531.

16. Morinville VD, Lowe ME, Ahuja $\mathrm{M}$, et al. Design and implementation of INSPPIRE. J Pediatr Gastroenterol Nutr 2014;59:360-364.

17. Charlson M, Szatrowski TP, Peterson J, Gold J. Validation of a combined comorbidity index. J Clin Epidemiol 1994;47:1245-1251.

18. Banks PA, Bollen TL, Dervenis C, et al; Acute Pancreatitis Classification Working Group. Classification of acute pancreatitis-2012: revision of the Atlanta classification and definitions by international consensus. Gut 2013;62:102-111.

19. Dellinger EP, Forsmark CE, Layer P, et al; Pancreatitis Across Nations Clinical Research and Education Alliance (PANCREA).
Determinant-based classification of acute pancreatitis severity: an international multidisciplinary consultation. Ann Surg 2012;256:875-880.

20. Marshall JC, Cook DJ, Christou NV, Bernard GR, Sprung CL, Sibbald WJ. Multiple organ dysfunction score: a reliable descriptor of a complex clinical outcome. Crit Care Med 1995;23:1638-1652.

21. Harris PA, Taylor R, Thielke R, Payne J, Gonzalez N, Conde JG. Research electronic data capture (REDCap) - a metadata-driven methodology and workflow process for providing translational research informatics support. J Biomed Inform 2009;42:377-381.

22. Mirtallo JM, Forbes A, McClave SA, Jensen GL, Waitzberg DL, Davies AR; International Consensus Guideline Committee Pancreatitis Task Force. International consensus guidelines for nutrition therapy in pancreatitis. JPEN J Parenter Enteral Nutr 2012;36:284-291.

23. Uhl W, Warshaw A, Imrie C, et al; International Association of Pancreatology. IAP guidelines for the surgical management of acute pancreatitis. Pancreatology 2002;2:565-573.

24. Working Group IAP/APA Acute Pancreatitis Guidelines. IAP/ APA evidence-based guidelines for the management of acute pancreatitis. Pancreatology 2013;13:e1-e15.

25. Wu BU, Bakker OJ, Papachristou GI, et al. Blood urea nitrogen in the early assessment of acute pancreatitis: an international validation study. Arch Intern Med 2011;171:669-676.

26. Kumar S, Ooi CY, Werlin S, et al. Risk factors associated with pediatric acute recurrent and chronic pancreatitis: lessons from INSPPIRE. JAMA Pediatr 2016;170:562-569.

27. Ting J, Wilson L, Schwarzenberg SJ, et al. Direct costs of acute recurrent and chronic pancreatitis in children in the INSPPIRE registry. J Pediatr Gastroenterol Nutr 2016;62:443-449.

28. van Santvoort HC, Besselink MG, Bakker OJ, et al; Dutch Pancreatitis Study Group. A step-up approach or open necrosectomy for necrotizing pancreatitis. N Engl J Med 2010;362:1491-1502.

29. Bakker OJ, van Brunschot S, van Santvoort HC, et al; Dutch Pancreatitis Study Group. Early versus on-demand nasoenteric tube feeding in acute pancreatitis. N Engl J Med 2014;371:19831993.

30. Fölsch UR, Nitsche R, Lüdtke R, Hilgers RA, Creutzfeldt W. Early ERCP and papillotomy compared with conservative treatment for acute biliary pancreatitis. The German Study Group on Acute Biliary Pancreatitis. N Engl J Med 1997;336:237-242.

31. James S, Rao SV, Granger CB. Registry-based randomized clinical trials-a new clinical trial paradigm. Nat Rev Cardiol 2015;12:312-316. 